TRANSACTIONS OF THE

AMERICAN MATHEMATICAL SOCIETY

Volume 172, October 1972

\title{
GROUPS OF LINEAR OPERATORS DEFINED BY GROUP CHARACTERS
}

BY

\author{
MARVIN MARCUS AND JAMES HOLMES( $\left.{ }^{1}\right)$
}

\begin{abstract}
Some of the recent work on invariance questions can be regarded as follows: Characterize those linear operators on $\operatorname{Hom}(V, V)$ which preserve the character of a given representation of the full linear group. In this paper, for certain rational characters, necessary and sufficient conditions are described that ensure that the set of all such operators forms a group $\mathfrak{L}$. The structure of $\mathfrak{L}$ is also determined. The proofs depend on recent results concerning derivations on symmetry classes of tensors.
\end{abstract}

1. Statements. Let $G$ be any subgroup of the full linear group $\mathrm{GL}(n, \mathrm{C})$ over the complex numbers, and let $\mathfrak{O}$ denote the linear closure of $G$ in the total matrix algebra $M_{n}(\mathrm{C})$. Let $K: G \rightarrow \mathrm{GL}(N, \mathrm{C})$ be a representation which is extended to a representation of the multiplicative semigroup of $\mathscr{U}$ in $M_{n}(C)$. Let $\mu_{K}(X)=\operatorname{tr} K(X)$ be the corresponding character. Next, let $\mathscr{L}(G, K)$ denote the multiplicative semigroup of all linear transformations $\mathfrak{T}: \mathfrak{U} \rightarrow \mathfrak{U}$ having the property that $\mathcal{T}$ preserves the character of the representation $K$; that is,

$$
\mu_{K}(\mathfrak{T}(X))=\mu_{K}(X), \quad X \in \mathfrak{X} .
$$

The two central questions which will concern us in this paper are:

(i) Under what circumstances is $\mathscr{L}(G, K)$ a group, i.e., under what circumstances is it true that if (1) holds, then $\mathcal{T}$ is nonsingular?

(ii) If $\mathcal{L}(G, K)$ is a group, then what is its structure?

Probably the first instance of a question of this kind was discussed by Frobenius [3] who proved that if $G=\mathrm{GL}(n, \mathrm{C})$, so that $\mathscr{U}=M_{n}(\mathrm{C})$, and if $K(X)=$ $\operatorname{det}(X)$, then $\mathscr{L}(G, K)$ is a group. He proved, in answer to question (ii), that for $\mathcal{T} \in \mathscr{L}(G, K)$ there exist fixed matrices $U$ and $V$ in $G L(n, C)$ such that

$$
\mathcal{T}(X)=U X V, \quad X \in M_{n}(\mathbf{C}), \quad \text { or } \quad \mathcal{T}(X)=U X^{T} V, \quad X \in M_{n}(\mathbf{C}),
$$

Received by the editors March 25, 1971.

AMS (MOS) subject classifications (1970). Primary 20G05, 15A15, 15A69; Secondary $20 \mathrm{~B} 05$.

Key words and phrases. Representations, characters, linear transformations, elementary divisors, symmetry classes of tensors, derivations on symmetry classes.

(1) The work of the first author was supported by the U. S. Air Force Office of Scientific Research under AFOSR 72-2164. The work of the second author was supported by the National Science Foundation under NSF GP-20632. 
where $\operatorname{det}(U V)=1\left(X^{T}\right.$ is the transpose of $\left.X\right)$.

A related problem was discussed by I. Schur [12]. Let $3 \leq m \leq n$ and $\mathcal{T}$ : $M_{n}(\mathrm{C}) \rightarrow M_{n}(\mathrm{C})$ be a linear transformation satisfying the following condition. For each $X \in M_{n}(C)$, the $m$ th order subdeterminants of $\mathcal{J}(X)$ are fixed linearly independent linear homogeneous functions of the $m$ th order subdeterminants of $X$. Schur proved that for such a $\mathfrak{T}$ there exist fixed matrices $U, V \in \mathrm{GL}(n, \mathrm{C})$ such that

$$
\mathcal{T}(X)=U X V, \quad X \in M_{n}(\mathbf{C}), \quad \text { or } \quad \mathcal{T}(X)=U X^{T} V, \quad X \in M_{n}(\mathbf{C}) .
$$

This problem can be reformulated in terms of the $m$ th Grassmann compound $C_{m}(X)$ of $X$. Let $S$ be a nonsingular linear transformation from $M_{\left(\begin{array}{c}n \\ m\end{array}\right)}(\mathrm{C})$ to itself. Characterize those linear transformations $\mathcal{T}$ on $M_{n}(C)$ which satisfy

$$
C_{m}(\mathcal{T}(X))=S\left(C_{m}(X)\right), \quad X \in M_{n}(\mathrm{C}) .
$$

This reformulation and a proof depending on more recent results appear in [9].

Let $P=\left[\begin{array}{ll}0 & 1 \\ 1 & 0\end{array}\right]$, and let $G$ be the group consisting of all $X \in \mathrm{GL}(2, \mathrm{C})$ for which $X^{*}=P X^{T} P$. Let $K(X)=\operatorname{det}(X)$. As can be readily verified, $\mathscr{L}(G, K)$ is isomorphic to the set of linear transformations mapping the real space $R^{4}$ into itself and holding fixed the quadratic form

$$
f(x)=x_{1}^{2}+x_{2}^{2}-x_{3}^{2}-x_{4}^{2}
$$

In [8] it is proved that $\mathscr{U}$ consists of all $X \in M_{2}(C)$ of the form

$$
\left[\begin{array}{ll}
z & w \\
\bar{w} & \bar{z}
\end{array}\right]
$$

and that $\mathscr{L}(G, K)$ consists of all $\mathcal{T}$ of the form

$$
\mathcal{T}(X)=U X V, \quad X \in M_{n}(\mathbf{C}), \quad \text { or } \quad \mathcal{T}(X)=U X^{T} V, \quad X \in M_{n}(\mathbf{C}),
$$

where $\operatorname{det}(U V)=1$, and $U^{*}=P U^{T} P, V^{*}=P V^{T} P$.

In [10] it is proved that $\mathcal{L}(G, K)$ is a group when $G=\mathrm{GL}(n, C)$ and $K(X)=$ $C_{m}(X)$ for $3<m \leq n$. In this instance, $\mu_{K}(X)=\operatorname{tr} C_{m}(X)$ is the $m$ th elementary symmetric function of the eigenvalues of $X$, or equivalently, the sum of all $\left(\begin{array}{l}n \\ m\end{array}\right) m$-square principal subdeterminants of $X$. In case $m<n$, the group $\mathcal{L}\left(\mathrm{GL}(n, \mathrm{C}), C_{m}\right)$ consists of precisely those linear transformations $\mathcal{T}$ of the form

$$
\mathcal{T}(X)=U X V, \quad X \in M_{n}(\mathrm{C}), \quad \text { or } \quad \mathcal{T}(X)=U X^{T} V, \quad X \in M_{n}(\mathbf{C}),
$$

where $U V=e^{i \phi} I_{n}$ and $m \phi \equiv O(2 \pi)$. This result was recently extended to include the case $m=3$ in [2].

Another minor modification of our problem occurs in [5]. Let $U(n, \mathrm{C})$ denote the subgroup of $\mathrm{GL}(n, \mathrm{C})$ consisting of all unitary matrices. Then the semigroup 
$\mathcal{L}$ of all linear transformations $\mathcal{T}$ on $M_{n}(\mathbf{C})$ which satisfy $\mathscr{T}(U(n, \mathbf{C})) \subset U(n, \mathbf{C})$ is a group. It is shown that $\mathfrak{T} \in \mathcal{L}$ if and only if there exist fixed matrices $U, V \in$ $U(n, \mathrm{C})$ such that

$$
\mathcal{T}(X)=U X V, \quad X \in M_{n}(\mathbf{C}), \quad \text { or } \quad \mathcal{T}(X)=U X^{T} V, \quad X \in M_{n}(\mathbf{C}) .
$$

Observe that $\mathscr{Q}(\mathscr{U}, K)$ is not always a group. Let $G=\mathrm{GL}(n, \mathrm{C})$ and $K(X)$ be the $m$ th Kronecker power $\Pi^{m}(X)$ of $X$ [14]. Then $\mu_{K}(X)=(\operatorname{tr}(X))^{m}$. The annihilator map $\mathcal{T}$ which sends each $X=\left(x_{i j}\right)$ into $\mathcal{T}(X)=\left(y_{i j}\right)$ where $y_{i j}=\delta_{i j} x_{i j}$ and which clearly belongs to $\mathcal{L}(\mathscr{U}, K)$, has no inverse.

In this paper we shall discuss problems (i) and (ii) for a certain class of rational representations of the multiplicative semigroup of $\mathfrak{U}$ which are in fact components of the $m$ th Kronecker product representation $\Pi^{m}(X)$. It is somewhat easier to state our results in an invariant setting.

Let $H$ be a subgroup of the symmetric group of degree $m$ and let $\chi$ be a character of $H$ of degree 1 . Let $V$ be an $n$-dimensional vector space over $\mathrm{C}$; let $U$ be any vector space over $\mathbf{C}$ and $\phi\left(v_{1}, \cdots, v_{m}\right)$ an $m$-multilinear function on the Cartesian product $X{ }_{1}^{m} V$ to $U$. Then $\phi$ is said to be symmetric with respect to $H$ and $X$ if

$$
\phi\left(v_{\sigma(1)}, \cdots, v_{\sigma(m)}\right)=\chi(\sigma) \phi\left(v_{1}, \cdots, v_{m}\right)
$$

holds for any $\sigma \in H$ and arbitrary vectors $\nu_{i} \in V$. A pair $(P, \nu)$ consisting of a vector space $P$ over $\mathrm{C}$ and a fixed m-multilinear function $\nu: X_{1}^{m} V \rightarrow P$, symmetric with respect to $H$ and $\chi$, is a symmetry class of tensors associated with $H$ and $\chi$ if

(i) $\langle\operatorname{mg} \nu\rangle=P$, i.e., the linear closure of the range of $\nu$ is $P$;

(ii) (universal factorization property) for any vector space $U$ over $\mathbf{C}$ and any m-multilinear function $\phi: X_{1}^{m} V \rightarrow U$, symmetric with respect to $H$ and $\chi$, there exists a unique linear function $b: P \rightarrow U$ such that $\phi=b \nu$; i.e., the following diagram is commutative.

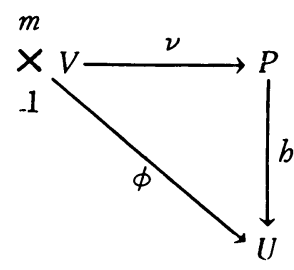

For any linear transformation $X: V \rightarrow V$ the preceding universal factorization property permits us to define a unique linear transformation $K(X): P \rightarrow P$, the induced transformation on $P$, which satisfies the following identity. For arbitrary vectors $v_{1}, \cdots, v_{m}$ in $V$ 


$$
K(X) \nu\left(v_{1}, \cdots, v_{m}\right)=\nu\left(X v_{1}, \cdots, X v_{m}\right) .
$$

By the spanning property of the range of $\nu$ (i.e., (i) above), (2) immediately implies that $K(X)$ is multiplicative and in fact if $m \leq n$ and $X \in \mathrm{GL}_{n}(V)$, the group of all linear bijections on $V$, then $K(X) \in \mathrm{GL}_{N}(P)$ where $N=\operatorname{dim} P$. If $G$ is any subgroup of $\mathrm{GL}_{n}(V)$ and $\mathfrak{U}$ is the linear closure of $G$ in $\operatorname{Hom}(V, V)$, we are thus in a position to discuss the structure of $\mathcal{L}(G, K)$, which for the class of representations $K(X)$ just defined depends on the group $H$ and the character $\chi$. If we identify $V$ with the space of $n$-tuples over $\mathrm{C}$, then of course $\mathrm{GL}_{n}(V)$ can be identified with $\mathrm{GL}(n, \mathrm{C})$ and we can ask for the structure of the semigroup $\mathcal{L}(G, K)$ for the preceding class of representations $K(X)$ of $\mathfrak{U}$.

Our main results follow.

Theorem 1. Let $\operatorname{dim} V=n, H \subset S_{m}, \chi$ a character of degree 1 on $H$. Let $(P, \nu)$ be the symmetry class associated with $H$ and $\chi$ and $X \rightarrow K(X)$ be a rep. resentation of $G=\mathrm{GL}_{n}(V)$ by induced transformations on $P$. If $m \leq n$ or $\chi \equiv 1$, then $\mathcal{L}\left(\mathrm{GL}_{n}(V), K\right)$ is a group if and only if $H \neq\{e\}$.

Theorem 2. Let $\operatorname{dim} V=n, H=S_{m}, m>1, \chi \equiv 1$. Let $G$ be a subgroup of $\mathrm{GL}_{n}(V)$. If the algebra $\mathcal{U}$ bas the property that the conjugate transpose $X *$ of each $X$ in $\mathfrak{X}$ is again in $\mathfrak{X}$, then $\mathfrak{Q}(G, K)$ is a group.

Theorem 3. In Theorem 1, take $H=S_{m}, m \geq 3$ and $\chi \equiv 1$. Let $\mathfrak{L}_{1}\left(\mathrm{GL}_{n}(V), K\right)$ denote the subgroup of $\mathscr{\&}\left(\mathrm{GL}_{n}(V), K\right)$ of those $\mathcal{T}: \operatorname{Hom}(V, V) \rightarrow \operatorname{Hom}(V, V)$ satisfying $\mathcal{T}\left(I_{V}\right)=\xi I_{V}$. Then $\stackrel{\complement}{1}_{1}\left(\mathrm{GL}_{n}(V), K\right)$ consists precisely of those linear transformations $\mathfrak{T}$ which bave the form

$$
\mathcal{I}(X)=\xi U^{-1} X U, \quad X \in \operatorname{Hom}(V, V),
$$

or

$$
\mathcal{T}(X)=\xi U^{-1} X^{T} U, \quad X \in \operatorname{Hom}(V, V)
$$

Theorem 4. In Theorem 1, take $H=A_{m} \subset S_{m}$ to be the alternating group, $m \geq 3$, and $\chi \equiv 1$. The group $\mathfrak{L}_{1}\left(\mathrm{GL}_{n}(V), K\right)$ consists precisely of those linear transformations $\mathcal{T}$ of the form (3) or (4).

Corollary 1. Let $\operatorname{dim} V=n, H=S_{m}, m>1, \chi \equiv 1$. If $G$ is the group of all $n \times n$ permutation matrices (so that $\mathfrak{U}$ is the algebra of generalized doubly stochastic matrices), then $\mathfrak{Q}(G, K)$ is a group.

Let $m$ and $n$ be positive integers. Let $Q_{m, n}$ (resp. $G_{m, n}$ ) denote the set of all strictly increasing (resp. nondecreasing) sequences of length $m$ chosen from the set $\{1,2, \cdots, n\}$. If $f\left(\lambda_{1}, \ldots, \lambda_{n}\right)$ is a polynomial symmetric in the indeter- 
minates $\lambda_{1}, \cdots, \lambda_{n}$ and $X \in \operatorname{Hom}(V, V)$, we shall denote by $f(X)$ the value of $f$ at the eigenvalues of $X$. For $m \geq 1$, let $b_{m}\left(\lambda_{1}, \cdots, \lambda_{n}\right)$ denote the $m$ th completely symmetric polynomial

$$
b_{m}\left(\lambda_{1}, \cdots, \lambda_{n}\right)=\sum_{a \in G_{m, n}} \prod_{t=1}^{m} \lambda_{\alpha(t)} ;
$$

and let $k_{m}\left(\lambda_{1}, \cdots, \lambda_{n}\right)$ denote the symmetric polynomial

$$
\begin{aligned}
k_{m}\left(\lambda_{1}, \ldots, \lambda_{n}\right) & =b_{m}\left(\lambda_{1}, \ldots, \lambda_{n}\right)+\sum_{\alpha \in Q_{m, n}} \prod_{t=1}^{m} \lambda_{a(t)} \\
& =b_{m}\left(\lambda_{1}, \ldots, \lambda_{n}\right)+E_{m}\left(\lambda_{1}, \ldots, \lambda_{n}\right),
\end{aligned}
$$

where $E_{m}\left(\lambda_{1}, \cdots, \lambda_{n}\right)$ is the $m$ th elementary symmetric function of $\lambda_{1}, \cdots, \lambda_{n}$ when $m \leq n$ and 0 if $m>n$.

Corollary 2. Let $m \geq 3$. Any linear transformation $\mathfrak{T}: \operatorname{Hom}(V, V) \rightarrow$ $\operatorname{Hom}(V, V)$ satisfying $\mathcal{T}\left(I_{V}\right)=\xi I_{V}$ and $b_{m}(\mathcal{T}(X))=b_{m}(X), X \in \operatorname{Hom}(V, V)$, has the form (3) or (4).

Corollary 3. Let $m \geq 3$. Any linear transformation $\mathfrak{T}: \operatorname{Hom}(V, V) \rightarrow$ $\operatorname{Hom}(V, V)$ satisfying $\mathcal{T}\left(I_{V}\right)=\xi I_{V}$ and $k_{m}(\mathcal{T}(X))=k_{m}(X), X \in \operatorname{Hom}(V, V)$, has the form (3) or (4).

We conjecture that in fact $\mathscr{L}_{1}\left(\mathrm{GL}_{n}(V), K\right)=\mathscr{L}\left(\mathrm{GL}_{n}(V), K\right)$ in Theorems 3 and 4. This amounts to showing that if $\mathcal{T}^{n} \mathrm{GL}_{n}(V) \rightarrow \mathrm{GL}_{n}(V)$ and $\mu_{K}(\mathcal{T}(X))=\mu_{K}(X)$ holds for all $X \in \operatorname{Hom}(V, V)$, then $\mathcal{T}\left(I_{V}\right)=\xi I_{V}$ where $\xi^{m}=1$.

2. Partial derivations. In [6] the standard notion of a derivation on a tensor algebra [4] is extended to higher order derivations on a general symmetry class $(P, \nu)$. We shall further extend the idea of a derivation induced by a single linear transformation to partial derivations induced by two linear transformations [11].

Let $T$ and $S$ be in $\operatorname{Hom}(V, V)$ and let $r+s=m$. For $\omega \in Q_{r, m}$ define

$$
\Pi_{\omega}(T, S)=\bigotimes_{i=1}^{m} X_{i}
$$

where $X_{i}=T$ for $i \epsilon \mathrm{mg} \omega$ and $X_{i}=S$ otherwise. In other words (5) is the tensor product of the linear transformations $T$ and $S$ in which $T$ appears in positions numbered $\omega$ and $S$ appears elsewhere. The linear transformation (5) acts on $\bigotimes_{1}^{m} V$, which of course is the symmetry class associated with $H=\{e\}$. Define

$$
\delta_{r, s}(T, S)=\sum_{\omega \in Q_{r, m}} \Pi_{\omega}(T, S)
$$


In order to simplify subsequent notation we make the following convention. Let $f: Q_{r, m} \times Q_{s, m} \rightarrow R$ be any function into a set $R$ having an associative addition. We shall let $\Sigma^{\prime} f(\omega)$ denote the summation of $f(\omega, \gamma)$ over all sequences $\omega \epsilon$ $Q_{r, m}, \gamma \in Q_{s, m}$ such that $\mathrm{mg} \omega \cap \mathrm{mg} \gamma=\varnothing$. Next, define

$$
M\left(X_{1}, \cdots, X_{m}\right)=\sum_{\phi \in S_{m}} X_{\dot{\phi}(1)} \otimes \cdots \otimes X_{\phi(m)} .
$$

Then it is easy to show that

$$
M_{r, s}(T, S)=r ! s ! \delta_{r, s}(T, S)
$$

where $M_{r, s}(T, S)$ denotes

$$
M(\overbrace{T, \cdots, T}^{r}, \frac{s}{S, \cdots, S}) .
$$

It is also a standard fact concerning symmetry classes that if the symmetry operator associated with $H$ and $\chi$ (a linear transformation on $\otimes_{1}^{m} V$ ) is defined by

$$
\tau_{\chi}=\frac{1}{|H|} \sum_{\sigma \in H} \chi(\sigma) \sigma
$$

$\left(\sigma\left(v_{1} \otimes \ldots \otimes v_{m}\right)=v_{\sigma-1}(1) \otimes \ldots \otimes v_{\sigma-1}(m)\right.$, then the pair $(P, \nu), P=\operatorname{rrig} \tau_{\chi}$ $c \otimes_{1}^{m} V, \nu\left(v_{1}, \cdots, v_{m}\right)=\tau_{\chi}\left(v_{1} \otimes \ldots \otimes v_{m}\right)$ is the symmetry class associated with $H$ and $\chi$. It is also easy to show that the transformation $M_{r, s}(T, S)$ satisfies $M_{r, s}(T, S)_{\sigma}=\sigma M_{r, s}(T, S)$ for all $\sigma \in S_{m}$; hence any symmetry class is an invariant subspace of $M_{r, s}(T, S)$. But then in view of (6), each symmetry class is an invariant subspace of $\delta_{r, s}(T, S)$.

We define the $(r, s)$ partial derivation associated with $T$ and $S$ on $(P, \nu)$ to be the restriction of $\delta_{r, s}(T, S)$ to the invariant subspace $P$. We denote this by $\Omega_{r, s}(T, S)$. The reason for calling $\Omega_{r, s}(T, S)$ the $(r, s)$ partial derivation on $(P, \nu)$ is the following formula:

$$
K\left(x_{1} T+x_{2} S\right)=\sum_{r+s=m} x_{1}^{r} x_{2}^{s} \Omega_{r, s}(T, S) .
$$

In order to verify (7) we compute that

$$
\begin{aligned}
& K\left(x_{1} T+x_{2} S\right) \nu\left(v_{1}, \cdots, v_{m}\right)=\nu\left(\left(x_{1} T+x_{2} S\right) v_{1}, \ldots,\left(x_{1} T+x_{2} S\right) v_{m}\right) \\
& (8) \quad=\sum_{r+s=m} x_{1}^{r} x_{2}^{s} \sum^{\prime} \nu\left(\cdots, T v_{\omega(1)}, \cdots, S v_{\gamma(1)}, \ldots, T v_{\omega(r)}, \ldots, S v_{\gamma(s)}, \ldots\right),
\end{aligned}
$$

where in the inside summand on the right side of (8) the $T$ occurs in precisely the positions numbered $\omega$ and the $S$ in positions numbered $\gamma$. On the other hand, 
$\Omega_{r, s}(T, S) \nu\left(v_{1}, \cdots, v_{m}\right)=\delta_{r, s}(T, S) \tau_{\chi}\left(v_{1} \otimes \cdots \otimes v_{m}\right)=\tau_{\boldsymbol{\chi}} \delta_{r, s}(T, S)\left(v_{1} \otimes \cdots \otimes v_{m}\right)$

$$
\begin{aligned}
& =r_{x} \sum^{\prime}\left(\cdots \otimes T v_{\omega(1)} \otimes \cdots \otimes S v_{\gamma(1)} \otimes \cdots \otimes T v_{\omega(r)} \otimes \cdots \otimes S v_{\gamma(s)} \otimes \cdots\right) \\
& =\sum^{\prime} \nu\left(\ldots, T v_{\omega(1)}, \cdots, S v_{\gamma(1)}, \cdots, T v_{\omega(r)}, \cdots, S v_{\gamma(s)}, \cdots\right) .
\end{aligned}
$$

Replacing (9) in (8) we have (7).

We observe a number of elementary facts concerning the partial derivation $\Omega_{r, s}(T, S)$ :

(i) If $X$ and $Y$ are in $\operatorname{Hom}(V, V)$, then

$$
K(X) \Omega_{r, s}(T, S) K(Y)=\Omega_{r, s}(X T Y, X S Y) .
$$

This follows immediately from (7).

(ii) If $V$ is a unitary space, then $\otimes_{1}^{m} V$ is also a unitary space. Thus there is a natural inner product induced on the symmetry class $(P, \nu)$ associated with $H$ and $\chi$. Moreover, if $T^{*}$ is the conjugate dual of $T \in \operatorname{Hom}(V, V)$, then the conjugate dual of $\Omega_{r, s}(T, S)$ with respect to the induced inner product in $(P, \nu)$ is

$$
\Omega_{r, s}(T, S)^{*}=\Omega_{r, s}\left(T^{*}, S^{*}\right)
$$

(iii) $\Omega_{1, m-1}(T, S)$ is linear in $T$ and $\Omega_{m-1,1}(T, S)$ is linear in $S$.

A somewhat more combinatorially involved description is necessary to itemize the eigenvalues of $\Omega_{r, s}(T, S)$. In order to describe a basis for an arbitrary symmetry class associated with $H$ and $\chi$, we regard the elements of $H$ as permutations acting on the functions (i.e., sequences) in $\Gamma_{m, n}=Z_{n}^{Z_{m}}$, where $Z_{q}=$ $\{1,2, \cdots, q\}$ and for $\sigma \in H, a \in \Gamma_{m, n}$,

$$
\sigma((\alpha))(t)=a \cdot\left(\sigma^{-1}(t)\right), \quad t \in Z_{m} .
$$

Let $\Delta$ denote a system of distinct representatives for the orbits in $\Gamma_{m, n}$ induced by $H$, and let $\bar{\Delta}$ denote the set of all of those elements $a \in \Delta$ for which the character $\chi$ is identically 1 on the stabilizer subgroup $H_{a}=\{\sigma \in H: \sigma(\alpha)=\alpha\}$. Let $n(\alpha)=\left|H_{\alpha}\right| \cdot$ It is routine to verify that if $\left\{e_{1}, \ldots, e_{n}\right\}$ is a basis of $V$, then the decomposable elements $\nu\left(e_{\alpha(1)}, \cdots, e_{a(m)}\right), \alpha \in \bar{\Delta}$ form a basis for $P$. In fact, if $\left\{e_{1}, \cdots, e_{n}\right\}$ is an orthonormal (hereafter abbreviated o.n.) basis of $V$, then the $|\bar{\Delta}|$ decomposable elements $(|H| / n(\alpha))^{1 / 2} \nu\left(e_{\alpha(1)}, \cdots, e_{\alpha(m)}\right)$ form an o.n. basis for $P$ with respect to the induced inner product in $\otimes_{1}^{m} V$ defined by

$$
\left(x_{1} \otimes \cdots \otimes x_{m}, y_{1} \otimes \cdots \otimes y_{m}\right)=\prod_{i=1}^{m}\left(x_{i}, y_{i}\right) \text {. }
$$


If we choose the system of distinct representatives $\Delta$ so that each sequence $a \epsilon \Delta$ is lowest in lexicographic order in the orbit in which it lies, then it is easy to see that $Q_{m, n} \subset \bar{\Delta}$ and $G_{m, n} \subset \Delta$, whatever the group $H$ and character $\chi$ may be [7].

If we use the fact that for any pair $(T, S)$ of commuting linear transformations there exists a common triangular o.n. basis, then it is not difficult to prove the. following [11]:

(iv) If $S T=T S$ and the eigenvalues of $T$ and $S$ are $\lambda_{1}, \cdots, \lambda_{n}$ and $\kappa_{1}, \cdots, \kappa_{n}$ respectively, then after a suitable reordering of the $\kappa_{i}$ 's, the eigenvalues of $\Omega_{r, s}(T, S)$ are the numbers

$$
\sum^{\prime} \prod_{i=1}^{r} \lambda_{\alpha \omega(i)} \prod_{j=1}^{s} \kappa_{a \gamma(j)}, \quad \alpha \in \bar{\Delta} .
$$

In particular, if $r=1$ and $s=m-1$, then the eigenvalues of $\Omega_{1, m-1}(T, S)$ are

$$
\sum_{t=1}^{m} \lambda_{a(t)} \prod_{j=t}^{m} \kappa_{a(j)}, \quad \alpha \in \bar{\Delta} .
$$

Some additional combinatorial maneuvering will be required: if $\alpha \epsilon \Gamma_{m, n}$ and $1 \leq t \leq n$, then let $m_{t}(\alpha)$ denote the number of integers $i$ in $\{1,2, \ldots, m\}$ for which $\alpha(i)=t$; i.e., $m_{t}(\alpha)$ is the multiplicity of occurrence of $t$ in the range of $a$. More generally, if $p_{1}+\cdots+p_{r}=n$ is a partition of $n$ into positive parts, we define

$$
\eta_{t}(\alpha)=\sum_{j=P_{t-1}+1}^{P_{t}} m_{j}(\alpha)
$$

where $P_{t}=p_{1}+\cdots+p_{t}$; i.e., $\eta_{t}(\alpha)$ is the number of times any integer $k$ satisfying $P_{t-1}<k \leq P_{t}$ occurs in the range of $a$. We can write the eigenvalues (13) of $\Omega_{1, m-1}(T, S)$ in a form somewhat more suitable for our subsequent computations. Suppose that the eigenvalues of $T$ are given by

$$
\lambda_{1}=\cdots=\lambda_{P_{1}}=l_{1} ; \lambda_{P_{1}+1}=\cdots=\lambda_{P_{2}}=l_{2} ; \cdots ; \lambda_{P_{r-1}+1}=\cdots=\lambda_{P_{r}}=l_{r}
$$

where the numbers $l_{t}$ are distinct. Suppose, moreover, that $f(x)$ is an arbitrary scalar polynomial and $S=f(T)$. In this case the ordering (14) of the eigenvalues of $T$ induces a corresponding ordering of the eigenvalues $\kappa_{1}, \ldots, \kappa_{n}$ of $S$, i.e.,

$$
\kappa_{1}=\cdots=\kappa_{P_{1}}=k_{1} ; \kappa_{P_{1}+1}=\cdots=\kappa_{P_{2}}=k_{2} ; \cdots ; \kappa_{P_{r-1}+1}=\cdots=\kappa_{n}=k_{r}
$$

Since the ploynomial $f(x)$ can be chosen arbitrarily, it follows that the numbers $k_{t}$ may be chosen arbitrarily. Regarding the $k_{t}$ as momentarily all different from zero, we see that the eigenvalues (13) become 


$$
\begin{aligned}
\sum_{t=1}^{m} \lambda_{\alpha(t)} \prod_{j \neq t}^{m} \kappa_{\alpha(j)} & =\sum_{t=1}^{m} \frac{\lambda_{\alpha(t)}}{\kappa_{\alpha(t)}} \prod_{j=1}^{m} \kappa_{\alpha(j)}=\sum_{t=1}^{m} \frac{\lambda_{\alpha(t)}}{\kappa_{\alpha(t)}} \prod_{j=1}^{n} \kappa_{j}^{m_{j}(a)} \\
& =\sum_{t=1}^{n} m_{t}(\alpha) \frac{\lambda_{t}}{\kappa_{t}} \prod_{j=1}^{n} \kappa_{j}^{m_{j}(a)}=\sum_{t=1}^{r} \eta_{t}(a) \frac{l_{t}}{k_{t}} \prod_{j=1}^{r} k_{j}^{\eta_{j}(a)} \\
& =\sum_{t=1}^{r} \eta_{t}(\alpha) l_{t} k_{t}^{\eta_{t}(a)-1} \cdot \prod_{j \neq t}^{r} k_{j}^{\eta_{j}(\alpha)} .
\end{aligned}
$$

If we interpret $0^{0}$ as 1 , then (15) holds even when some of the numbers $k_{t}$ are zero. We now have the following formula for the trace of $\Omega_{1, m-1}(T, S)$ :

$$
\begin{aligned}
\operatorname{tr} \Omega_{1, m-1}(T, S) & =\sum_{a \in \bar{\Delta}} \sum_{t=1}^{r} \eta_{t}(\alpha) l_{t} k_{t}^{\eta_{t}(\alpha)-1} \prod_{j \neq t}^{r} k_{j}^{\eta_{j}^{(\alpha)}} \\
& =\sum_{t=1}^{r} l_{t}\left(\sum_{a \in \bar{\Delta}} \eta_{t}(\alpha) k_{t}^{\eta_{t}(\alpha)-1} \prod_{j \neq t}^{r} k_{j}^{\eta_{j}^{(\alpha)}}\right) .
\end{aligned}
$$

\section{Proofs.}

Lemma 1. Let $G$ be any subgroup of $\mathrm{GL}_{n}(V)$ and $\mathcal{T} \in \mathcal{L}(G, K)$. Then if $A$ $\epsilon \operatorname{ker} \mathfrak{T}$,

$$
\operatorname{tr} \Omega_{1, m-1}(A, X)=0, \quad X \in \mathfrak{U} .
$$

Proof. Let $x_{1}, x_{2}$ be indeterminates over C. From (7) we have

$$
K\left(x_{1} A+x_{2} X\right)=\sum_{r=0}^{m} x_{1}^{r} x_{2}^{m-r} \Omega_{r, m-r}(A, X) .
$$

Thus, if $\mathcal{T}(A)=0$ we have

$$
\begin{aligned}
\operatorname{tr} \sum_{r=0}^{m} x_{1}^{r} x_{2}^{m-r} \Omega_{r, m-r}(A, X) & =\mu_{K}\left(x_{1} A+x_{2} X\right)=\mu_{K}\left(\mathcal{T}\left(x_{1} A+x_{2} X\right)\right) \\
& =\mu_{K}\left(\mathcal{T}\left(x_{2} X\right)\right)=\mu_{K}\left(x_{2} X\right)=x_{2}^{m} \mu_{K}(X)
\end{aligned}
$$

If we equate coefficients in (18) we obtain

$$
\operatorname{tr} \Omega_{r, m-r}(A, X)=0, \quad r=1,2, \cdots, m,
$$

and hence (17) follows.

Proof of Theorem 1. Assume $H \neq\{e\}$ and let $A \in$ ker $\mathcal{T}$. We show that $A=$ 0 . By (10) we can assume that (17) holds for all $X$ and that $A$ is in Jordan normal form.

Lemma 2. If every elementary divisor of $A$ is linear and $A \in \operatorname{ker} \mathcal{T}$, then $A=0$. 
Proof. By (iii) of $\$ 2$, (17) becomes

$$
\sum_{i=1}^{n} a_{i i} \operatorname{tr} \Omega_{1, m-1}\left(E_{i i}, X\right)=0, \quad X \in M_{n}(\mathrm{C}) .
$$

We first assume that $\chi \equiv 1$. The eigenvalues of $E_{i i}$ are $\lambda_{i}=1$ and $\lambda_{j}=0$, $j \neq i$; while those of $E_{k k}$ are $\kappa_{k}=1$ and $\kappa_{j}=0, j \neq k$. Hence

$$
\operatorname{tr} \Omega_{1, m-1}\left(E_{k k}, E_{k k}\right)=\sum_{a \in \Delta} \sum_{t=1}^{m} \kappa_{a(t)} \prod_{j \neq t}^{m} \kappa_{a(j)}=\sum_{t=1}^{m} \kappa_{k}^{m}=m,
$$

because the only term which survives in the inner summation in (20) is the term corresponding to that $a$ for which $a(1)=a(2)=\cdots=a(m)=k$. We remark that this sequence is always in $\bar{\Delta}$ since, as we remarked, $G_{m, n} \subset \bar{\Delta}$ when $\chi \equiv 1$. If $i \neq k$, we again compute that

$$
\operatorname{tr} \Omega_{1, m-1}\left(E_{i i}, E_{k k}\right)=\sum_{\alpha \in \bar{\Lambda}} \sum_{t=1}^{m} \lambda_{a(t)} \prod_{j \neq t} \kappa_{\alpha(j)}=\sum_{\beta \in \bar{\Delta}} 1 \cdot 1^{m-1}
$$

where the inner summation in (21) is over precisely those $\beta \in \bar{\Delta}$ for which $m_{k}(\beta)=$ $m-1$ and $m_{i}(\beta)=1$. Once again, since $G_{m, n} \subset \bar{\Delta}$, such sequences exist and we let $p_{i k}$ denote their number. We assert that $p_{i k}$ is independent of the pair $(i, k)$; for if $P$ is an arbitrary permutation matrix we have from (10)

$$
p_{i k}=\operatorname{tr} \Omega_{1, m-1}\left(E_{i i}, E_{k k}\right)=\operatorname{tr} \Omega_{1, m-1}\left(P^{T} E_{i i} P, P^{T} E_{k k} P\right) .
$$

Obviously if $i \neq k$, we can choose $P$ so that $P^{T} E_{i i} P=E_{i}{ }^{\prime}{ }^{\prime}$ and $P^{T} E_{k k} P=$ $E_{k}{ }^{\prime}{ }_{k}$ for any preassigned distinct integers $i^{\prime}, k^{\prime}$. We set $p$ equal to the common value of the $p_{i k}$. We next assert that $p<m$, for since $H \neq\{e\}$, there must exist at least two sequences $\alpha \neq \beta$ in the same $H$-orbit for which $m_{k}(\alpha)=m_{k}(\beta)=$ $m-1$ and $m_{i}(\alpha)=m_{i}(\beta)=1$. Thus there are at most $m-1$ elements of $\bar{\Delta}$ with that property. If we set $X$ successively equal to $E_{k k}, k=1,2, \cdots, n$, in (19) we obtain the following system of linear equations:

$$
m a_{i i}+\sum_{k+i} p a_{k k}=0, \quad 1 \leq i \leq n .
$$

Since $p<m$, the coefficient matrix in this system is nonsingular and we conclude that $a_{i i}=0$ for $1 \leq i \leq n$. Thus since the elementary divisors of $A$ are linear we conclude $A=0$.

We now consider the case in which $\chi \not \equiv 1$. If $x$ is an indeterminate, then since $E_{i i}$ and $I_{n}+x E_{11}$ commute we have from (13) that

$$
\begin{aligned}
\operatorname{tr} \Omega_{1, m-1}\left(E_{i i}, I_{n}+x E_{11}\right) & =\sum_{a \in \bar{\Delta}} \sum_{t=1}^{m} \lambda_{a(t)} \prod_{j \neq t} \kappa_{a(j)} \\
& =\sum_{a \in \bar{\Delta}} \prod_{j=1}^{n} \kappa_{j}^{m_{j}(\alpha)} \sum_{t=1}^{n} m_{t}(\alpha) \frac{\lambda_{t}}{\kappa_{t}}
\end{aligned}
$$


where $\lambda_{1}, \ldots, \lambda_{n}$ are again the eigenvalues of $E_{i i}$ and $\kappa_{1}, \cdots, \kappa_{n}$ are the eigenvalues of $I_{n}+x E_{11}$. In case $i=1$, the right side of (22) becomes

$$
\sum_{\alpha \in \bar{\Delta}} m_{1}(\alpha)(1+x)^{m_{1}(\alpha)-1}
$$

which we denote by $\phi_{1}(x)$. If $i>1$, we see that the value of (22) (which is of course the same for $i=2,3, \ldots, n$ ) is

$$
\phi_{2}(x)=\sum_{\alpha \in \bar{\Delta}} m_{2}(\alpha)(1+x)^{m_{1}(\alpha)}
$$

With $X=I_{n}+x E_{11}$ in (19) we have from (23) and (24) that

$$
\begin{aligned}
& a_{11} \phi_{1}(x)+\phi_{2}(x) \sum_{i=2}^{n} a_{i i} \\
& =a_{11} \sum_{a \in \Xi} m_{1}(\alpha)(1+x)^{m_{1}(\alpha)-1}+\left(\sum_{i=2}^{n} a_{i i}\right)\left(\sum_{a \in \Delta} m_{2}(\alpha)(1+x)^{m_{1}(\alpha)}\right)=0 .
\end{aligned}
$$

We observe that

$$
q_{j}=\sum_{\alpha \in \Xi} m_{j}(\alpha)
$$

is independent of $j$ and we denote this common value by $q$. A proof of this can be based on the fact that the eigenvalues of $K(X)$ are

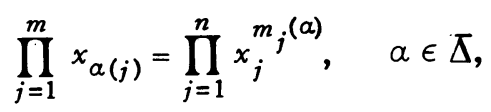

where $x_{1}, \cdots, x_{n}$ are the eigenvalues of $X$. Thus

$$
\operatorname{det}(K(X))=\prod_{\alpha \in \bar{\Delta}} \prod_{j=1}^{n} x_{j}^{m_{j}(\alpha)}=\prod_{j=1}^{n} x_{j}{ }_{j}{ }
$$

Since $\operatorname{det}\left(K\left(P^{T} X P\right)\right)=\operatorname{det}(K(X))$ for any permutation matrix $P$, we conclude that

$$
\prod_{j=1}^{n} x_{j}^{q_{j}}=\prod_{j=1}^{n} x_{\sigma(j)}^{q_{j}}, \quad \sigma \in S_{n}
$$

And since $x_{1}, \cdots, x_{n}$ are arbitrary, $q_{1}=q_{2}=\cdots=q_{n}$. For example, it is easy to compute that

$$
q=q_{1}=\sum_{a \in Q_{m, n}} m_{1}(\alpha)=\left(\begin{array}{c}
n-1 \\
m-1
\end{array}\right) ;
$$

and since $K(X)$ is the familiar $m$ th compound martix $C_{m}(X)$ we see that

$$
\operatorname{det} C_{m}(X)=\prod_{j=1}^{m} x_{j}^{q}=(\operatorname{det}(X))^{\left(\begin{array}{l}
n-1 \\
m-1
\end{array}\right)},
$$


and we have as a corollary to our computations the well-known Sylvester-Franke theorem [1]. For $x=0$, (25) becomes $q \operatorname{tr} A=0$ and hence $\operatorname{tr} A=0$; we therefore rewrite (25) as

$$
\left(\phi_{1}(x)-\phi_{2}(x)\right) a_{11}=0
$$

Clearly,

$$
\operatorname{deg} \phi_{1}(x)=\max _{a \in \bar{\Delta}} m_{1}(\alpha)-1
$$

Now $\chi \not \equiv 1$, and thus there exists $\beta \in \bar{\Delta}$ and $1<j \leq n$ such that

$$
j \in \operatorname{rng} \beta, \quad m_{1}(\beta)=\max _{\alpha \in \bar{\Delta}} m_{1}(\alpha),
$$

for otherwise $\max _{a \in \bar{\Delta}} m_{1}(\alpha)=m$ and $\alpha=(1,1, \cdots, 1) \in \bar{\Delta}$. But the stabilizer of this $a$ is obviously all of $H$ and it would follow that $\Sigma_{\sigma \in H} \chi(\sigma) \neq 0$ so that $\Sigma_{\sigma \in H} \chi(\sigma)=|H|$. This can only happen if $\chi \equiv 1$, since $\chi$ is a character of degree 1. Hence

$$
\operatorname{deg} \phi_{1}(x)=m_{1}(\beta)-1 \text { and } \operatorname{deg} \phi_{2}(x)=m_{1}(\beta),
$$

so that $\phi_{1}(x)-\phi_{2}(x) \neq 0$. From (27) it follows that $a_{11}$ (and hence any $a_{i i}$ ) is 0 . Thus $A=0$, completing the proof of Lemma 2. We can now remove the condition that $A$ has linear elementary divisors.

Lemma 3. If $A \in \operatorname{ker} \mathcal{T}$, then $A=0$.

Proof. From (11) we know that

$$
\Omega_{1, m-1}(A, X)^{*}=\Omega_{1, m-1}\left(A^{*}, X^{*}\right) .
$$

Since (17) holds for all $X$, we have $\operatorname{tr} \Omega_{1, m-1}\left(A^{*}, X\right)=0$ and hence tr $\Omega_{1, m-1}\left(A \pm A^{*}, X\right)=0$. Now $A \pm A^{*}$ is normal and hence has linear elementary divisors. Applying Lemma 2, we conclude that $A \pm A^{*}=0$ and therefore $A=0$.

We have proved that if $H \neq\{e\}$ and $m \leq n$ or $\chi \equiv 1$, then any $\mathcal{T} \epsilon$ $\mathcal{L}\left(\mathrm{GL}_{n}(V), K\right)$ is nonsingular; since we have observed that this set obviously forms a semigroup, it is in fact a group. Conversely, if $H=\{e\}$, then the symmetry class $P$ is just the $m$ th tensor space $\bigotimes_{1}^{m} V$ and $K(X)=\Pi^{m}(X)$. As we saw in $\S 1, \mathcal{L}(G, K)$ is not a group. This completes the proof of Theorem 1 .

Proof of Theorem 2. We observe that if $A \in \mathfrak{U}$ and $f(x)$ is any scalar polynomial, then $f(A) \in \mathfrak{U}$. The eigenvalues of $\Omega_{1, m-1}(A, f(A))$ are given by (13) where $\lambda_{1}, \ldots, \lambda_{n}$ are the eigenvalues of $A$ and $\kappa_{1}, \ldots, \kappa_{n}$ are the eigenvalues of $f(A)$. Moreover, it is clear that the values of $f(x)$ may be arbitrarily assigned at the distinct eigenvalues of $A$. 
Since $H=S_{m}$ and $\chi \equiv 1$, the symmetry class $P$ is precisely the $m$ th completely symmetric space, usually denoted by $V^{(m)}$ [4], and the sequence set $\bar{\Delta}$ is precisely $G_{m, n}$. Once again the problem is to show that if $\mathcal{T}(A)=0$, then $A=0$.

We have from Lemma 1 and formula (16) that

$$
\sum_{t=1}^{r} l_{t}\left(\sum_{a \in \bar{\Delta}} \eta_{t}(\alpha) k_{t}^{\eta_{t}^{(\alpha)-1}} \prod_{j \neq t}^{r} k_{j}^{\eta_{j}^{(\alpha)}}\right)=0
$$

in which the distinct eigenvalues of $A$ are described in (14) and the numbers $k_{1}, \ldots, k_{r}$ may be chosen arbitrarily. The first part of the proof is devoted to showing that all the eigenvalues of $A$ are equal (i.e., that $r=1$ ). Assume then that $r>1$. For a fixed $t$, set $k_{t}=1$ and $k_{j}=0$ for $j \neq t$; observe that the coefficient of $l_{t}$ in (28) is

$$
m \sum_{a \in \bar{\Delta}, \eta_{t}(a)=m} 1=m\left(\begin{array}{c}
p_{t}+m-1 \\
m
\end{array}\right),
$$

where the indicated binomial coefficient is just a count of the total number of sequences $\alpha$ in $G_{m, n}$ such that $\operatorname{rng} \alpha \subset\left\{P_{t-1}+1, \ldots, P_{t}\right\}$. The coefficient of $l_{s}, s \neq t$, in (28) is

$$
\sum_{\alpha \in \bar{\Delta}, \eta_{s}(\alpha)=1, \eta_{t}(\alpha)=m-1} 1
$$

which is a count of the total number of nondecreasing sequences $\alpha$ of length $m$ which have the property that $\mathrm{mg}$ a contains precisely 1 integer in the interval $\left[P_{s-1}+1, P_{s}\right]$ and $m-1$ integers in $\left[P_{t-1}+1, P_{t}\right]$. Since there are precisely

$$
\left(\begin{array}{c}
p_{t}+m-2 \\
m-1
\end{array}\right) \cdot p_{s}
$$

such sequences, the value of (29) is (30). Thus (28) for the choice $k_{t}=1, k_{j}=0$ for $j \neq t$ is

$$
l_{t} m\left(\begin{array}{c}
p_{t}+m-1 \\
m
\end{array}\right)+\sum_{s \neq t}^{r} l_{s} p_{s}\left(\begin{array}{c}
p_{t}+m-2 \\
m-1
\end{array}\right)=0
$$

or

$$
l_{t}\left(p_{t}+m-1\right)+\sum_{s \neq t}^{r} l_{s} p_{s}=0
$$

Consider the system of homogeneous linear equations for $l_{1}, \ldots, l_{r}$ obtained by setting $t=1,2, \cdots, r$ in (31). By setting $k_{1}=\cdots=k_{r}=1$ in (28) we obtain the following additional condition on the l's: 


$$
\sum_{t=1}^{r} l_{t}\left(\sum_{\alpha \in \Delta} \eta_{t}(\alpha)\right)=0
$$

Now by (26)

$$
\sum_{\alpha \in \bar{\Delta}} \eta_{t}(\alpha)=\sum_{\alpha \in \bar{\Delta}} \sum_{j=P_{t-1}^{+1}}^{P_{t}} m_{j}(\alpha)=\sum_{j=P_{t-1}+1}^{P_{t}} q_{j}=p_{t} \cdot q .
$$

Thus (32) becomes

$$
\sum_{s=1}^{r} l_{s} p_{s}=0
$$

Combining the system (31) with (33) we have $(m-1) l_{t}=0, t=1, \cdots, r$, and thus $l_{t}=0, t=1, \cdots, r$, contradicting the fact that the $l_{1}, \ldots, l_{r}$ are distinct. Hence $r=1$. In other words, the condition

$$
\operatorname{tr} \Omega_{1, m-1}(A, X)=0, \quad X \in \mathfrak{U},
$$

implies that all the eigenvalues of $A$ are equal. If we set $X=I_{n}$ in (34) we then see that

$$
\begin{aligned}
& \operatorname{tr} \Omega_{1, m-1}\left(A, I_{n}\right)=\sum_{a \in \bar{\Delta}} \sum_{t=1}^{m} \lambda_{a(t)}=\sum_{a \in \bar{\Delta}} \sum_{t=1}^{n} m_{t}(\alpha) \lambda_{t} \\
& =\sum_{t=1}^{n} q \cdot \lambda_{t}=q \operatorname{tr} A=0 .
\end{aligned}
$$

Thus we conclude that if $\operatorname{tr} \Omega_{1, m-1}(A, X)=0, X \in \mathfrak{U}$, then all the eigenvalues of $A$ are zero. By repeating precisely the same argument as we gave in Lemma 3, we can conclude that the eigenvalues of both of the normal matrices $A \pm A^{*}$ are zero and hence $A=0$. This completes the proof of Theorem 2 .

The proof of Corollary 1 is now obvious, since the conjugate transpose of a generalized doubly stochastic matrix is a matrix of the same kind.

Proof of Theorem 3. We are assuming that $\mathcal{T}: \operatorname{Hom}(V, V) \rightarrow \operatorname{Hom}(V, V)$ satisfies

$$
\mu_{K}(\mathscr{T}(X))=\mu_{K}(X), \quad X \in \operatorname{Hom}(V, V) .
$$

Since $\mu_{K}(\xi X)=\xi^{m} \mu_{K}(X)$ it is clear that we may assume $\mathcal{T}\left(I_{n}\right)=I_{n}$. From (35) we have

$$
\operatorname{tr} K\left(\mathcal{T}\left(I_{n}+x X\right)\right)=\mu_{K}\left(\mathcal{T}\left(I_{n}+x X\right)\right)=\mu_{K}\left(I_{n}+x X\right)=\operatorname{tr} K\left(I_{n}+x X\right) .
$$

From (7) with $T=I_{n}, S=X, x_{1}=1, x_{2}=x$ we have 


$$
\sum_{r=0}^{m} x^{r} \operatorname{tr} \Omega_{m-r, r}\left(I_{n}, \mathcal{J}(X)\right)=\sum_{r=0}^{m} x^{r} \operatorname{tr} \Omega_{m-r, r}\left(I_{n}, X\right)
$$

and thus

$$
\operatorname{tr} \Omega_{m-r, r}\left(I_{n}, \mathcal{T}(X)\right)=\operatorname{tr} \Omega_{m-r, r}\left(I_{n}, X\right)
$$

Let $\kappa_{1}, \cdots, \kappa_{n}$ be the eigenvalues of $X$. Then as we know from (12), the eigenvalues of $\Omega_{m-r, r}\left(I_{n}, X\right)$ are

$$
\sum^{\prime} \prod_{j=1}^{r} \kappa_{a \gamma(j)}, \quad \alpha \in \Delta
$$

where we recall that the prime indicates that the summation is over all $\gamma \in Q_{r, m}$. But the expression (37) is precisely the $t$ th elementary symmetric function of $\kappa_{a(1)}, \cdots, \kappa_{a(m)}, E_{r}\left(\kappa_{a(1)}, \cdots, \kappa_{a(m)}\right)$. Thus

$$
\operatorname{tr} \Omega_{m-r, r}\left(I_{n}, X\right)=\sum_{a \in G_{m, n}} E_{r}\left(\kappa_{a(1)}, \cdots, \kappa_{a(m)}\right),
$$

and an elementary induction argument shows that the right-hand side of (38) is precisely

$$
\left(\begin{array}{c}
m+n-1 \\
m-r
\end{array}\right) b_{r}\left(\kappa_{1}, \cdots, \kappa_{n}\right)
$$

where $b_{r}$ denotes the $r$ th completely symmetric polynomial in the $\kappa$ 's. From (36) and (39) it follows that

$$
b_{r}\left(\kappa_{1}^{\prime}, \cdots, \kappa_{n}^{\prime}\right)=b_{r}\left(\kappa_{1}, \cdots, \kappa_{n}\right), \quad r=1,2, \cdots, m,
$$

where the $\kappa_{1}^{\prime}, \ldots, \kappa_{n}^{\prime}$ are the eigenvalues of $\mathcal{T}(X)$. But by Wronski's relations [13] we know that the completely symmetric polynomials $b_{1}, \ldots, b_{m}$ form an integral polynomial basis for the space of all integral homogeneous symmetric polynomials of degree $m$. Thus it follows that

$$
E_{r}\left(\kappa_{1}^{\prime}, \cdots, \kappa_{n}^{\prime}\right)=E_{r}\left(\kappa_{1}, \cdots, \kappa_{n}\right), \quad r=1,2, \ldots, m .
$$

In other words, we have proved that the $r$ th elementary symmetric function of the eigenvalues of both $X$ and $\mathcal{T}(X)$ are equal, $r=1,2, \ldots, m$; and we are in a position to apply a theorem of Marcus and Purves [10] (recently extended by Beasley [2]) which states that any such transformation must have one of the two forms indicated in (3) or (4). This completes the proof of Theorem 3.

The proof of Corollary 2 is an immediate consequence of Theorem 3. For, the eigenvalues of $K(X)$ when $H=S_{m^{\prime}}, \chi \equiv 1, P=V^{(m)}$ are the $\left(\begin{array}{c}n+m-1 \\ m\end{array}-1\right)$ 
homogeneous products

$$
\prod_{t=1}^{n} \lambda_{t}^{m_{t}^{(\alpha)}}, \quad \alpha \in G_{m, n}
$$

and hence

$$
\mu_{K}(X)=\operatorname{tr} K(X)=\sum_{\alpha \in G_{m, n}} \prod_{t=1}^{n} \lambda_{t}^{m_{t}(\alpha)}=b_{m}\left(\lambda_{1}, \ldots, \lambda_{n}\right) .
$$

Proof of Theorem 4. Once again we can assume that $\mathcal{T}\left(I_{n}\right)=I_{n^{*}}$ Our first task is to determine the structure of the $\bar{\Delta}$ set for $H=A_{m}$ and $\chi \equiv 1$. There are two cases to consider: $m \leq n$ and $m>n$. Let $\omega \in \Gamma_{m, n^{*}}$. If the integers in rng $\omega$ are distinct, then let $\alpha$ be the sequence such that $\mathrm{mg} \alpha=\operatorname{mg} \omega$ and $\alpha(1)<\alpha(2)<$ $\ldots<\alpha(m)$, and let $\beta$ be the sequence such that $\operatorname{rng} \beta=\operatorname{mg} \omega$ and $\beta(1)<\beta(2)<$ $\ldots<\beta(m-2)<\beta(m)<\beta(m-1)$. It is clear that $\alpha$ and $\beta$ lie in distinct $A_{m}{ }^{-o r-}$ bits and that any other sequence $\gamma \in \Gamma_{m, n}$ for which $\operatorname{mg} \gamma=\operatorname{mg} \omega$ is in the same $A_{m}$-orbit with either $\alpha$ or $\beta$. Thus each such sequence $\omega \in \Gamma_{m, n}$ gives rise to two elements in $\bar{\Delta}$, namely $\alpha$ and $\beta$. On the other hand, if $\omega \epsilon \Gamma_{m, n}$ satisfies $m_{t}(\omega) \geq 2$ for some $1 \leq t \leq n$, then it is obvious that there exists a sequence $a$ in $G_{m, n}$ in the same $A_{m}$-orbit with $\omega$. Thus in the case $m \leq n$, the system of distinct representatives may be chosen to be $\bar{\Delta}=G_{m, n} \cup Q_{m, n}^{\prime}$, where $Q_{m, n}^{\prime}$ consists of precisely those sequences $\beta$ for which $\beta(1)<\beta(2)<\cdots<\beta(m-2)<$ $\beta(m)<\beta(m-1)$. In the case that $m>n$, then it is clear that any sequence $\omega \epsilon$ $\bar{\Delta}$ lies in the same $A_{m}$-orbit with a sequence $a \in G_{m, n}$, so that we can choose $\bar{\Delta}=G_{m, n}$. In order to deal with both cases at once we will let $Q_{m, n}^{\prime}=\varnothing$ if $m>n$.

Precisely as in (36) we see that if $\mathcal{T} \in \mathscr{L}_{1}\left(\mathrm{GL}_{n}(V), K\right)$, then

$$
\operatorname{tr} \Omega_{m-r, r}\left(I_{n}, \mathcal{J}(X)\right)=\operatorname{tr} \Omega_{m-r, r}\left(I_{n}, X\right), \quad X \in M_{n}(\mathrm{C}) .
$$

The eigenvalues of $\Omega_{m-r, r}\left(I_{n}, X\right)$ are precisely the numbers

and thus

$$
E_{r}\left(\kappa_{\alpha(1)}, \cdots, \kappa_{\alpha(m)}\right), \quad \alpha \in \bar{\Delta},
$$

$$
\begin{aligned}
\operatorname{tr} \Omega_{m-r, r}\left(I_{n}, X\right) & =\sum_{\alpha \in \bar{\Delta}} E_{r}\left(\kappa_{\alpha(1)}, \cdots, \kappa_{\alpha(m)}\right) \\
& =\sum_{\alpha \in G_{m, n}} E_{r}\left(\kappa_{\alpha(1)}, \cdots, \kappa_{\alpha(m)}\right)+\sum_{a \in Q_{m, n}^{\prime}} E_{r}\left(\kappa_{\alpha(1)}, \cdots, \kappa_{\alpha(m)}\right) \\
& =\left(\begin{array}{c}
n+m-1 \\
m-r
\end{array}\right) b_{r}\left(\kappa_{1}, \cdots, \kappa_{n}\right)+\left(\begin{array}{c}
n-r \\
m-r
\end{array}\right) E_{r}\left(\kappa_{1}, \cdots, \kappa_{n}\right) .
\end{aligned}
$$


In case $m>n$, the elementary symmetric function does not appear in (41) and it is clear from (40) that

$$
\begin{aligned}
b_{r}(\mathfrak{T}(X)) & =\left(\begin{array}{c}
n+m-1 \\
m-r
\end{array}\right)^{-1} \operatorname{tr} \Omega_{m-r, r}\left(I_{n}, \mathcal{J}(X)\right) \\
& =\left(\begin{array}{c}
n+m-1 \\
m-r
\end{array}\right)^{-1} \operatorname{tr} \Omega_{m-r, r}\left(I_{n}, X\right) \\
& =b_{r}(X), \quad r=1,2, \ldots, m ; X \in M_{n}(\mathrm{C}) .
\end{aligned}
$$

Hence by Corollary 2, $\mathcal{T}$ has the required form. On the other hand, if $3 \leq m \leq n$, then using Wronski's relations we have

$$
\begin{aligned}
& \operatorname{tr} \Omega_{m-1,1}\left(I_{n}, X\right)=a E_{1}(X), \\
& \operatorname{tr} \Omega_{m-2,2}\left(I_{n}, X\right)=b b_{2}(X)+c E_{2}(X) \\
& =b\left(E_{1}^{2}(X)-E_{2}(X)\right)+c E_{2}(X) \\
& =(c-b) E_{2}(X)+b E_{1}^{2}(X), \\
& \operatorname{tr} \Omega_{m-3,3}\left(I_{n}, X\right)=d b_{3}(X)+e E_{3}(X) \\
& =d\left(E_{1}^{3}(X)-2 E_{1}(X) E_{2}(X)+E_{3}(X)\right)+e E_{3}(X) \\
& =(d+e) E_{3}(X)-2 d E_{1}(X) E_{2}(X)+d E_{1}^{3}(X),
\end{aligned}
$$

where $a=\left(\begin{array}{c}m+n-1 \\ m-1\end{array}\right)+\left(\begin{array}{c}n-1 \\ m-1\end{array}\right), b=\left(\begin{array}{c}n+m-1 \\ m-2\end{array}\right), c=\left(\begin{array}{c}n-2 \\ m-2\end{array}\right), d=\left(\begin{array}{c}n+m-1 \\ m-3\end{array}\right)$ and $e=\left(\begin{array}{c}n-3 \\ m-3\end{array}\right)$. Observe that $d+e>0, c-b \neq 0, a \neq 0$; thus the relations (42) allow us to express $E_{3}(X)$ as a polynomial in $\operatorname{tr} \Omega_{m-r, r}\left(I_{n}, X\right), r=1,2,3$. It follows from (40), then, that $E_{3}(\mathcal{T}(X))=E_{3}(X), X \in M_{n}(\mathrm{C})$ and hence we can conclude as before that $\mathfrak{T}$ has the required form. This completes the proof of Theorem 4.

By similar arguments Corollary 3 follows from Theorem 4. For, the eigenvalues of $K(X)$ when $H=A_{m}, X \equiv 1$ are the numbers

$$
\prod_{t=1}^{n} \lambda^{m_{t}^{(\alpha)}}, \quad \alpha \in G_{m, n} \cup Q_{m, n}^{\prime}
$$

and hence

$$
\begin{aligned}
\mu_{K}(X) & =\operatorname{tr} K(X)=\sum_{\alpha \in G_{m, n}} \prod_{t=1}^{n} \lambda_{t}^{m_{t}(\alpha)}+\sum_{\alpha \in Q_{m, n}} \prod_{t=1}^{n} \lambda_{t}^{m_{t}(\alpha)} \\
& =k_{m}\left(\lambda_{1}, \cdots, \lambda_{n}\right) .
\end{aligned}
$$




\section{REFERENCES}

1. A. C. Aitken, Determinants and matrices, Oliver and Boyd, Edinburgh; Interscience, New York, 1962, pp. 90-110.

2. L. B. Beasley, Linear transformations on matrices: The invariance of the third elementary symmetric function, Canad. J. Math. 22 (1970), 746-752. MR 42 \#3100.

3. G. Frobenius, Über die Darstellung der endlichen Gruppen durch lineare Substitutionen. I, S.-B. Preuss. Akad. Wiss. Berlin 1897, 994-1015.

4. W. H. Greub, Multilinear algebra, Die Grundlehren der math. Wissenschaften, Band 136, Springer-Verlag, New York, 1967. MR 37 \#222.

5. M. Marcus, All linear operators leaving the unitary group invariant, Duke Math. J. 26 (1959), 155-163. MR 21 \#54.

6. - Spectral properties of higher derivations on symmetry classes of tensors, Bull. Amer. Math. Soc. 75 (1969), 1303-1307. MR 41 \#245.

7. M. Marcus and W. R. Gordon, The structure of bases in tensor spaces, Amer. J. Math. 92 (1970), 623-640. MR $42 \# 7684$.

8. M. Marcus and N. A. Kahn, A note on a group defined by a quadratic form, Canad. Math. Bull. 3 (1960), 143-148. MR 23 \#A1653.

9. M. Marcus and F. May, On a theorem of I. Schur concerning matrix transformations, Arch. Math. 11 (1960), 401-404. MR 24 \#A134.

10. M. Marcus and R. Purves, Linear transformations on algebras of matrices: The invariance of the elementary symmetric functions, Canad. J. Math. 11 (1959), 383-396. MR $21 \# 4167$.

11. R. Merris, A generalization of the associated transformation, Linear Algebra and Appl. 4 ( 1971), 393-406.

12. I. Schur, Einige Bemerkungen zur Determinantentheorie, S.-B. Preuss. Akad. Wiss. Berlin 25 (1925), Satz II, 454-463.

13. H. W. Turnbull, Theory of equations, Oliver and Boyd, Edinburgh; Interscience, New York, 1952, pp. 71-72.

14. J. H. M. Wedderburn, Lectures on matrices, Amer. Math. Soc. Colloq. Publ., vol. 17, Amer. Math. Soc., Providence, R. I., 1934, 79pp.

DEPARTMENT OF MATHEMATICS, UNIVERSITY OF CALIFORNIA, SANTA BARBARA, CALIFORNIA 93106

DEPARTMENT OF MATHEMATICS, WESTMONT COLLEGE, SANTA BARBARA, CALIFORNIA 93108 